\title{
PRODUCING AND MEASURING SMALL ELECTRON BUNCHES *
}

\author{
X.J. Wang \\ Brookhaven Accelerator Test Facility, National Synchrotron Light Source \\ Brookhaven National Laboratory \\ Upton, NY 11973,USA
}

\begin{abstract}
Significant progress has been made in the last few years in ultra-short and high-brightness electron beam production and measurements. For many applications, such as laser and high-frequency RF (W-band) accelerators, and high-gain harmonic generation free electron laser, electron beams with bunch length and transverse emittance smaller than optical wavelength are required. The status of such small electron beam production around the world will be briefly reviewed. The experimental results of small electron beam production using photocathode RF gun with magnetic bunch compression system, and emittance growth in the compressor will be discussed. We will also discuss various techniques for characterizing such small electron beam, specially RF kicker cavity for photocathode RF gun based linac, and using coherent radiation for bunch length measurement. The analysis of the kilo-Ampere 10 fs electron beam generation at the ATF will be presented .
\end{abstract}

\section{INTRODUCTION}

One of the fundamental phenomena during the interaction between the charge particle bunches and electromagnetic fields is the distribution of the charge particle beam will be modified by the electromagnetic fields, which in turn will affect the field properties. The basic task for accelerator science is to learn how to produce and control the charge particle beam. We have taken granted for a long time the fact, magnets are commonly used to confine and modified the transverse profile of the charge particle beam. The challenge in controlling the longitudinal distribution (bunching or debunching) of the particle beam is that, the speed of the charge particle becomes more difficult to manipulate as the beam energy increase due to the relativistic effect. We are also limited in manipulating the electron bunches in low energy because of the space charge effect. The common phrase in our daily life, such as "time is flying" reflects the simple fact that, we have great difficulty in catching the fast objects, such as the electron bunches, and measuring its bunch distribution. These difficult subjects, the status of producing and

\footnotetext{
"Work supported by U.S D.O.E contract DE-AC02-98CH10886

"Email: xwang@bnl.gov
}

measuring small electron bunches will be discussed in this report.

Generally speaking, smallness in producing and measuring the electron bunches longitudinal distribution is relative to two physical parameters. One is the wavelength of the acceleration fields employed to accelerate the electron bunches, small electron bunches implies that, the phase spread of the electron bunch is a small fraction of $2 \pi$. For a S-band (2856 Mhz) linac, electron bunch length smaller than $10 \mathrm{ps}$ is needed. For a typical plasma based laser accelerator schemes, the wavelength of the plasma wave is on the order of $100 \mu \mathrm{m}$, so electron beam on the order of $10 \mu \mathrm{m}$ (30 fs) is needed in order to preserve the beam quality [1]. For Inverse Cerenkov, and Inverse Free Electron Laser (IFEL) accelerators using $\mathrm{CO}_{2}$ laser, the bunch length on the order of $1 \mu \mathrm{m}$ is preferred [2].

The second natural scale in measuring the small electron bunches is the transition time. Many dynamic process, such as phase transition and biological mutation occurs under room temperature on the time scale $\tau$,

$$
\tau \approx \eta / k T \approx 100 f_{s}
$$

In the following section, the status of the small bunches production and measurement is briefly reviewed. We will discuss the experimental results of short bunch production from photocathode RF gun based linac, and discuss RF kicker cavity for measuring small beam for FEL applications. We will present an initial analysis of producing kA 10 fs electron bunch at the Brookhaven Accelerator Test Facility (ATF).

\section{STATUS OF SMALL BUNCHES GENERATION AND MEASUREMT}

Short electron bunches production and measurement can be treated as two different aspects of the same problem. Significant progress were made in both fronts in the last decade[3]. One of the most important feature in this development is the mutual promotion between the production and measurement techniques in short bunch research. This is best exemplified by the development in using coherent radiation for bunch length measurement as electron bunches getting shorter. We will briefly review the small bunches production and measurement techniques for sub-picosecond electron bunches, and discuss emittance growth caused by the CSCF and CSR. 


\subsection{Short Bunches Production}

The shorter electron bunches can be produced in many ways, conceptually it can be classify into three categories and their combination. The three commonly used techniques in producing small electron bunches are:

Direct production: Using photocathode RF gun [4], plasma bases laser accelerator techniques(LILAC) [5-6], electron bunch from pico-second to femto-second can be produced directly. The primary issue in direct production in high brightness short electron bunches is the space charge effect, which varies as $1 / \gamma 3$. To produce subpicosecond short electron bunches directly with reasonable charge $\left(>10^{7}\right.$ electrons $)$ require high acceleration gradient to minimise the space charge effect. The coupling between the longitudinal and transverse space also plays critical role in direct producing subpicosecond electron bunches due to the divergence of the electron beam.

Selection:Taking advantage of electron beam longitudinal position dependency on the transverse position (dispersion), short bunches as part of a long electron bunch can be selected. This ideas was implemented at the BNL ATF to generate sub-picosecond slice beam, and to study the photocathode RF gun emittance compensation process[7]. Another example is the femto-second X-ray production at ALS [8], where small portion of the electron beam was first energy modulated by a femto-seconds laser, and energy modulated electron beam was selected in the dispersion region to produce femto-second X-ray.

Compression: For low energy electron beam, both ballistic[9] and $\alpha$-magnet[10] compressions were used to produce sub-picosecond electron bunches. In ballistic compression, faster (higher energy) electrons trail the slower electron, so both ends of the electron bunches move toward the centre of the bunch, which could be used to counter balance the bunch lengthening by space charge effect.

To overcome space charge effects, the most popular scheme to produce small electron bunches is magnetic compressor at higher energy. It takes advantage of the relativistic effect $\Delta \mathrm{v} / \mathrm{v}=\left(1 / \gamma^{2}\right) \Delta \mathrm{p} / \mathrm{p}$. Magnetic compressor is designed in such way that, the electrons path length inside the compressor is proportional to their energy. Electrons locate at the head of the bunch posses higher energy, travel longer path and falling behind. While electrons at tail catch up since they travel in shorter distance. So magnetic compressor must consist two parts. The electron bunches must be first energy modulated to produce the desired energy distribution, and energy modulated electron bunch is compressed when it passes through the magnetic system.

A typical chicane magnetic compressor consists of four equal strength dipole magnets. Using transport notation, the bunch length compression property is described by $R_{56}$ [11],

$$
\begin{aligned}
& R_{56}=\int\left(\frac{1}{\gamma^{2}}-\frac{D(s)}{R}\right) d s \approx-\int \frac{D(s)}{R} d s \\
&=2 \theta_{B}{ }^{2}\left(\Delta L+\frac{2}{3} L_{B}\right)
\end{aligned}
$$

Where $\theta_{\mathrm{B}}$ is the bending angle of the dipole magnets, and $\Delta \mathrm{L}$ is the separation between the magnets, and $\mathrm{L}_{\mathrm{B}}$ is the path length of the dipole magnet. The major advantage of 4-magnet chicane compressor is that, due to the symmetry of the arrangement, all higher order geometric effects are cancelled. The minimum bunch length produced by the compressor is determined by the ratio of the initial energy spread to the amplitude of the energy modulation.

Using a BNL ATF photocathode RF gun injection system and chicane magnetic compressor, University of Tokyo has successfully compressed a 13 ps, 250 pC charge electron bunch to less than 240 fs (FWHM) [12](Fig.1).

Non-linear and wake field in the chicane magnet compressor were extensively studied [13], the second order chromatic bunch lengthening is proportional to the first order term $R_{55}$, this limits on the correlated energy spread, and hence the final bunch length.

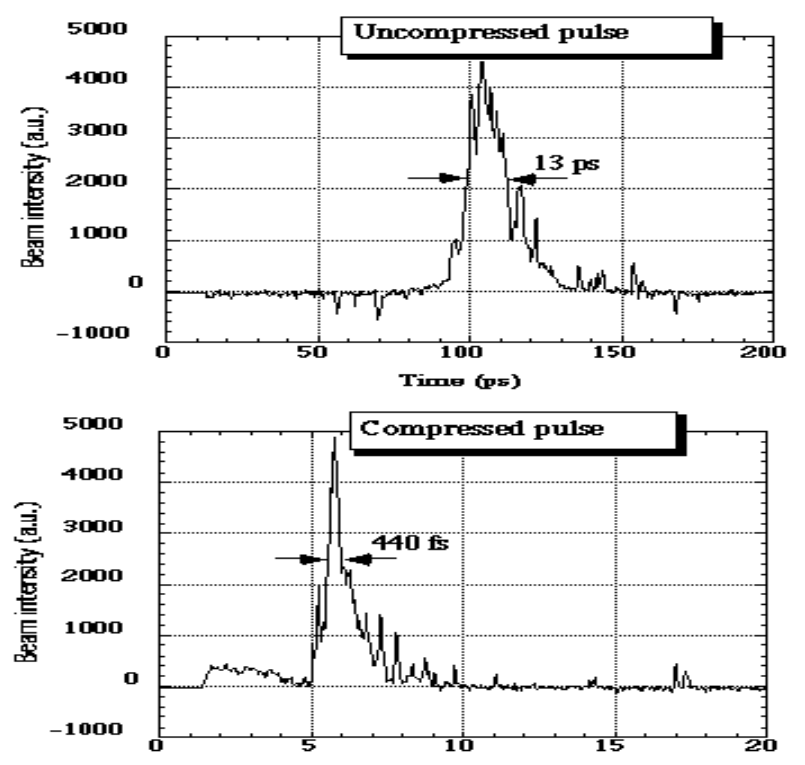

Figure 1: $250 \mathrm{pC}$ charge electron bunch is compressed to less than 240 fs after 370 fs streak camera resolution is considered.

Preserve the beam emittance is critical in any bunch compressor. Two energy independent effects could significantly increase the electron bunch transverse emittance for small electron bunches. One is so called Centrifugal Space Charge Force (CSCF), which is short range and caused by the curved trajectory of the electron bunches. The emittance growth by CSCF is [14], 


$$
\Delta \varepsilon^{C S C F}=0.38 N \alpha^{2} \frac{I_{p}}{I_{A}} \ln \left(\frac{h}{4 \sigma_{x}}\right) \frac{2 \sigma^{2}{ }_{x}}{3 \sigma_{z}}
$$

where $\mathrm{N}$ is the total number of the bending magnets, $\alpha$ is the bending angle, $I_{A}$ is the Alfven current, $I_{p}$ is the peak current, $\mathrm{h}$ is the dipole magnet chamber height, $\sigma_{\mathrm{z}}$ is the bunch length, and $\sigma_{x}$ is the bending plane beam spot size. The other is Coherent Synchrotron Radiation (CSR), which is long range, and can radiate significant amount of power. The emittance growth for CSR is [14],

$$
\Delta \varepsilon^{C S R}=0.5 N \alpha^{2} \frac{I_{p}}{I_{A}} \sigma_{x}\left(\frac{R}{\sigma_{z}}\right)^{1 / 3}
$$

where $\mathrm{R}$ is the bending radius of the dipole magnet. Using ATF 20 deg. double bend achromatic transport system, we have measured emittance growth due to CSCF and CSR for a $250 \mathrm{pC}$ charge[15]. Fig. 2 shows measured bunch length, and emittance growth as function of the electron beam spot size at the dipole. It agreed with the theoretical prediction, similar measurement for chicane magnet compressor was done recently at CERN[16].

\subsection{Small bunches measurement}

The other aspect of the small bunch physics is the techniques for measuring the bunch length. As electron bunches getting shorter, it demands new techniques to handle the new issues arise. Such as transverse form factor effect on coherent radiation from micro-bunched electron beam. There are many reviews on the bunch length measurement techniques recently $[17,18]$. We will concentrate on time domain and coherent radiation techniques in the rest of the section.

Streak camera is a single-shot time domain technique. The state of art streak camera is represented by FCS-200 streak camera manufactured by Hammmasu, it has demonstrated a 370 fs (FWHM) resolution (Fig.1). Using streak camera for electron beam bunch length measurement involves production and imaging the optical radiation the streak camera. Many effects could introduce significant error in sub-picosecond measurement, such as dispersion of the optics; bandwidth of the filter [19] and the finite size of the source. Transition radiation is the choice for very short bunch length measurement because of its promptness. For short electron bunch length measurement, the synchronisation between the streak camera and accelerator RF system could significantly enhance the experiment. Space charge effect in the streak camera tube limit the dynamic range of the measurement.
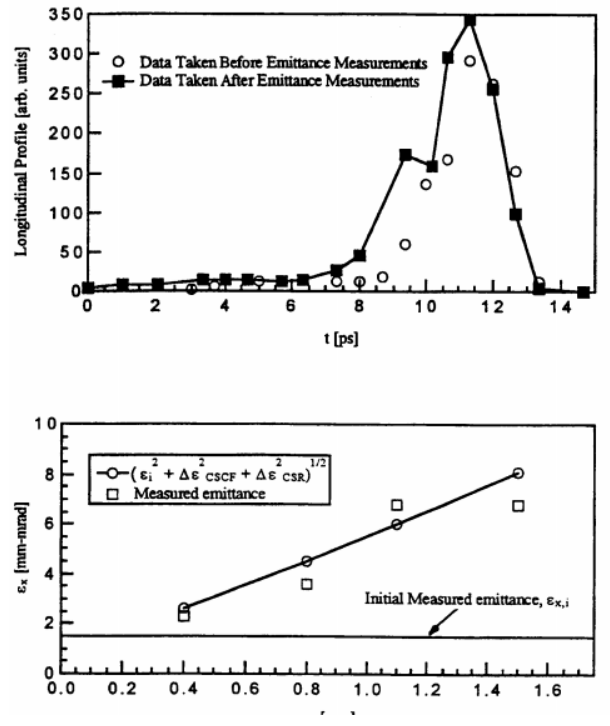

Figure 2: Emittance growth measurement for a $250 \mathrm{pC}$ charge bunch. Top is the bunch length measurement, bottom is the compression between the measurement and theory.

$$
\begin{gathered}
\frac{\Delta p_{y}}{p_{z}} \approx k \Delta z \\
\frac{\Delta p_{z}}{p_{z}} \approx k y \\
k=\frac{2 \pi}{\lambda} \frac{A}{\gamma} \sqrt{P Q}
\end{gathered}
$$

RF cavity operating in the $\mathrm{TM}_{110}$ mode(kicker cavity) is another time domain measurement device (Fig.3). It has been used in many laboratories for low energy beams. When electron bunches pass through the zero phase crossing, the amplitude of the kick effect can be described by [20],

Where $\Delta \mathrm{z}$ is the electron position inside the electron bunch, $\mathrm{k}$ is the kicker constant,

where $\mathrm{A}$ is constant related to the cavity dimension, $\lambda$ is the RF wavelength, $\mathrm{P}$ is the RF power, and $\mathrm{Q}$ is the quality factor of the cavity, and $\gamma$ is the relativistic factor.

For SASE X-ray FEL and linear collider applications, electron bunch compression usual occurs at hundreds $\mathrm{MeV}$ or a few GeV. It seems RF kicker cavity has little application for high energy short bunches measurement. 
Carefully examine Eq.(6), using shorter wavelength RF power source $(\lambda)$, or using superconducting RF cavity (high $\mathrm{Q}$, such as B-factory crab cavity), femto-seconds resolution can be realised by the RF kicker cavity with a high resolution beam profile monitor. Good resolution of the RF kicker cavity come from two important sources. One is the space charge effect which is negligible at high energy, second is small geometric emittance of the electron beam. RF kicker cavity enjoys many advantages over streak camera. It can be self calibrated, single shot to produce longitudinal profile of the electron bunches. It can be used for non-destructive timing jitter measurement of the electron bunches combining with beam position monitor, and can measure the longitudinal phase space of the electron beam in the dispersive beam line[20].

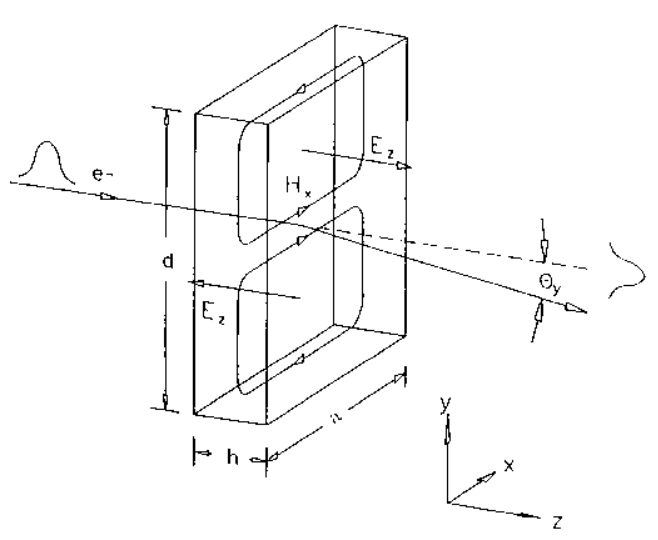

Figure 3: Schematic of the RF kicker cavity.

As electron beam bunch length getting shorter, the shielding effect from beam pipe is reduced, so coherent radiation from small electron bunches getting stronger. The total radiation power generated by a bunch of $\mathrm{N}$ charge particles can be expressed as,

$$
\text { I tot }(\omega)=\mathrm{I}(\omega)[\mathrm{N}+\mathrm{N}(\mathrm{N}-1) \mathrm{F}(\omega)]
$$

Where $\mathrm{I}(\omega)$ is the single electron radiation power and,

$$
\mathrm{F}(\omega)=\int \mathrm{S}(\mathrm{r}) \mathrm{e}^{-2 \pi \omega \mathrm{r}} \mathrm{dr}=\mathrm{F}_{\mathrm{L}}(\omega) \mathrm{F}_{\mathrm{T}}(\omega)
$$

$F(\omega)$ is the Fourier transform of three-dimension beam distribution $\mathrm{S}(\mathrm{r})$, which can be further divided into transverse form factor $\mathrm{F}_{\mathrm{T}}(\omega)$ and longitudinal form factor $\mathrm{F}_{\mathrm{L}}(\omega)$. The first part of $\mathrm{Eq}(1)$ is the incoherent radiation and second part is coherent radiation. It is coherent radiation contains the bunch length information. Longitudinal form factor is none negligible only when radiation wavelength is comparable with the bunch length. After initial experimental observations of coherent radiation in Tohoku University of Japan and Cornell University [21,22], Fourier spectrometer using Michelson interferometer was successfully used at Stanford University [10] to measured hundred femto-second long electron pulse train. This techniques can be treated both in time domain and frequency domain, the width of the interferogram is the auto-correlation of the coherent radiation, which can be used directly to estimated the electron beam bunch length. The spectrum of the coherent radiation can be obtained by Fourier transform the interfrogram. The bunch length information from coherent radiation can be extracted using Kramers-Kronig relation [22]. Good agreement was observed between streak camera measurement and coherent radiation technique at University of Tokyo [12](Fig.4).

Interfrometric technique has demonstrated many advantages, such as simplicity, cost effective and no fundamental limit in its resolution. Recently, holographic Fourier spectroscopy technique was suggested [20] for single-shot measurement, and to eliminate mechanical constrain. The detector bandwidth and calibration of the measurement are two main challenges as electron bunch length getting shorter for coherent radiation technique.

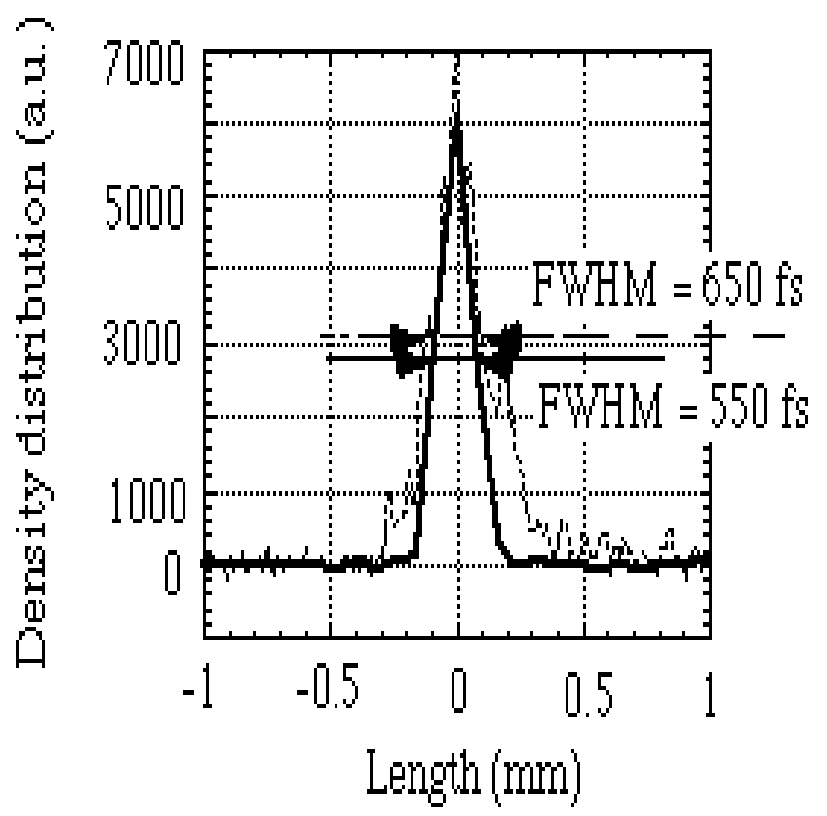

Figure 4: Reconstructed bunch distribution by the interferometry (solid curve) and the result by the streak camera (dashed curve).

\section{EXTREME SMALL ELECTRON BUNCH PRODUCTION AT THE ATF}

Photocathode RF gun not only can optimise the transverse emittance of the electron beam, it is also capable of control the longitudinal emittance of the electron beam. It is later that determine the final bunch length can be achieved in the compressor. The longitudinal emittance of the photoelectron beam can be expressed by:

$$
\begin{aligned}
\varepsilon_{\varphi} & =\sqrt{\left.<\phi^{2}><\left(\frac{\Delta p}{p}\right)^{2}>-<\phi \frac{\Delta p}{p}\right\rangle^{2}} \\
& \approx \frac{1}{2} \sigma_{\phi}{ }^{3} \cos \left(\phi_{0}\right)
\end{aligned}
$$


where $\sigma_{\phi}$ is the longitudinal phase spread of the photoelectron beam at the RF gun exit, $\phi_{0}$ is the photoelectron launch phase. Above equation shows that, photoelectron beam longitudinal emittance strongly depends on its bunch length at the RF gun exit. Fig.5 is the experimental results of the photoelectron beam bunch length as function of the RF gun phase produced by the 10 ps (FWHM) laser pulse. By optimising the RF gun phase, we were able to produce a 200 fs (FWHM) (330 fs full width) electron bunch with $40 \mathrm{pc}$ charge, the normalise emittance measured was $0.5 \mathrm{~mm}$-mrad. Combining with a magnetic compressors consist of undulator and chicane magnet, we can further reduce the electron bunch length. We will present a preliminary analysis how to produce a kilo-Ampere $10 \mathrm{fs}$ long electron bunch at the ATF.

Two recent developments at the ATF made it possible producing such short electron bunches, one the the linac energy upgrade to more than $70 \mathrm{MeV}$ [23], and other is the install undulator and chicane magnets for HGHG experiment [24]. The 10 fs long electron bunch will be produced in three stages. From Fig.5, we can produce a 20 pC charge, $30 \mathrm{MeV}, 100 \mathrm{fs}$ (FWHM) long electron bunch by reducing the RF gun operating phase. With recent ATF linac energy upgrade, first section of the ATF two sections of travelling linac will be able to produce $30 \mathrm{MeV}$ electron bunch. The second section will be phased in such way that, 0.5 to $1 \%$ correlated energy spread introduce to the $100 \mathrm{fs}$ long electron beam. This electron beam then was compressed first by the modulator undulator of the ATF HGHG experiment, whose $R_{56}$ is about $10 \mu \mathrm{m} / \%$. The under compressed beam is further compressed by the HGHG dispersion magnet with $\mathrm{R}_{56}$ equals to $35 \mu \mathrm{m} / \%$.

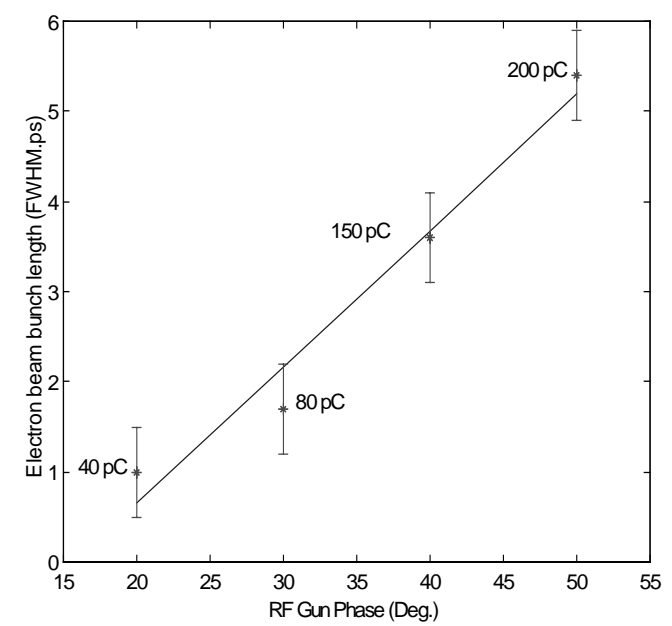

Figure 5: Photo-electron bunch length dependency on the RF gun phase.

The limiting factor of the final bunch length will be the electron beam divergence and space charge induced bunch lengthening. The bunch lengthening caused by the beam divergence can be calculated by:

$$
\Delta \lambda=\frac{1}{2} \int\left(x^{, 2}+y^{, 2}\right) d s
$$

To minimise the bunch lengthening, care must be taken to design the beam transport line. The space charge effect can be estimated by[25]:

$$
\Delta \lambda=\frac{2 Q c z^{2}}{I_{A} \gamma^{4} R L}
$$

where $\gamma$ is relativistic energy, $Q$ is the charge, $R$ and $L$ are transverse and longitudinal size of the beam, and $\mathrm{z}$ is the beam transport distance. Because of strong dependence on the beam energy $\gamma$, this effect is less than a few femtoseconds for a $30 \mathrm{MeV}$ beam.

\section{REFERENCES}

[1] T.C. Chiou and T. Katsouleas, Phys. Rev. Lett. 81, 3411 (1998).

[2]W.D. Kimura et al, To be published in the Proc. Of $8^{\text {th }}$ AAC workshop, Baltimore, Maryland (1998).

[3] Proceeding of Micro Bunches Workshop, AIP Conference Proceeding 367, edited by E.B. Blum, M. Dienes and J.B. Murphy (1995).

[4] L. Serafini et at, Nucl. Inst. Methods A 387, 305-314(1997).

[5] D. Umstadter at al, Phys. Rev. Lett. 76, 1285 (1995).

[6] E. Esarey at al, Phys. Rev. Lett. 79, 2682 (1998).

[7] X. Qiu et al, Phys. Rev. Lett. 76, 3723 (1996).

[8] A.A. Zholents et al, Phys. Rev. Lett. 76, 912 (1996).

[9] X. J. Wang et al, Phys. Rev. E 54, R3121 (1996).

[10] P. Kung at al, Phys. Rev. Lett. 73, 967 (1994).

[11]T.O. Raubenheimer et al, Proc. Of 1993 PAC, p.635-637 (1993).

[12]M. Uesaka et al, To be published in the Proc. Of $8^{\text {th }}$ AAC workshop, Baltimore, Maryland (1998).

[13] B.E. Carlsten, Nucl. Inst. Methods A 380, 505-516 (1996).

[14]B.E. Carlsten et al, Phys. Rev. E 51, 1453 (1995).

[15]X.J. Wang and D. Kehne, BNL-64646 (1997).

[16] H.H. Braun et al, submitted to Phys. Rev. Lett. , SLAC-PUB7758 (1999).

[17]A.H. Lumplin, FEL Chanllenges II, San Jose, Jan., 1999.

[18]D.X. Wang, Proc. Of PAC97, (1997).

[19] M. Uesaka et al, Nucl. Inst. Methods A 406, 371-379(1998).

[20]K.T. McDonald and D.P. Russell, Lecture Note in 343 published by Springer-Verlag, p.122-132(1988).

[21] T. Nakazato et al, Phys. Rev. Lett. 63, 1245 (1989).

[22] R. Lai and A.J. Sievers, Nucl. Inst. Methods A 397, 221231(1997).

[23] X.J. Wang et al, these proceeding.

[24] L.H. Yu et al, these proceeding.

[25] T.C. Katsouleas et al , IEEE Transactions on Plasma Science 24, 443(1996). 